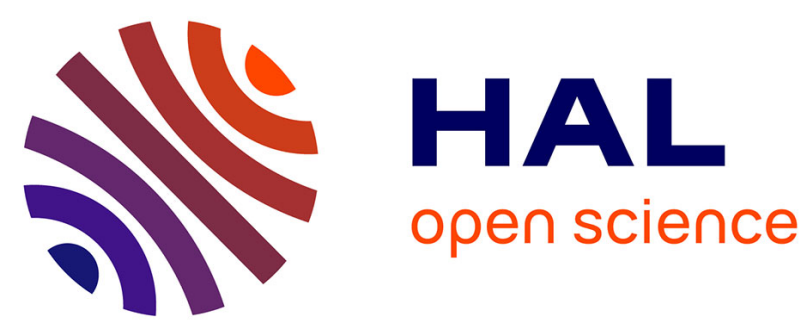

\title{
Simplified Analysis of HARQ Cooperative Networks Using Finite-State Markov Chains
}

\author{
Faton Maliqi, Francesca Bassi, Pierre Duhamel, Ilir Limani
}

\section{To cite this version:}

Faton Maliqi, Francesca Bassi, Pierre Duhamel, Ilir Limani. Simplified Analysis of HARQ Cooperative Networks Using Finite-State Markov Chains. European Signal Processing Conference (EUSIPCO), Eurasip, Aug 2017, Kos, Greece. 10.23919/eusipco.2017.8081561 . hal-01535664

\section{HAL Id: hal-01535664 \\ https://hal-centralesupelec.archives-ouvertes.fr/hal-01535664}

Submitted on 27 Apr 2020

HAL is a multi-disciplinary open access archive for the deposit and dissemination of scientific research documents, whether they are published or not. The documents may come from teaching and research institutions in France or abroad, or from public or private research centers.
L'archive ouverte pluridisciplinaire HAL, est destinée au dépôt et à la diffusion de documents scientifiques de niveau recherche, publiés ou non, émanant des établissements d'enseignement et de recherche français ou étrangers, des laboratoires publics ou privés. 


\title{
Simplified Analysis of HARQ Cooperative Networks Using Finite-State Markov Chains
}

\author{
Faton Maliqi ${ }^{\dagger \ddagger}$, Francesca Bassi ${ }^{\dagger \S}$, Pierre Duhamel ${ }^{\dagger}$, Ilir Limani ${ }^{\ddagger}$ \\ † L2S - CNRS-CentraleSupelec-Université Paris-Sud, Gif-sur-Yvette, France \\ ${ }^{\S}$ ESME-Sudria, Ivry-sur-Seine, France \\ $\ddagger$ University of Prishtina, Faculty of Electrical and Computer Engineering, Prishtina, Kosovo
}

\begin{abstract}
This paper considers the analysis of communication protocols in wireless networks implementing both cooperation and Hybrid Automatic Repeat reQuest (HARQ) for Type I decoder and Type II decoder with Chase Combining. Using an example of a three-node network, we show that the communication protocol can be modeled using Finite State Markov Chains. This model efficiently predicts the performance of the system. However, the complexity depends on the number of states, which increases very fast as the protocol gets more sophisticated. We then derive a simplified model using state aggregation, and obtain a compact description which can be used to predict the performance with a reduced complexity. Moreover, we show that the simplified model describes a probabilistic communication protocol on the same network. Monte Carlo simulations show that the theoretical predictions match the simulated performance.
\end{abstract}

\section{INTRODUCTION}

In wireless communications the difficult propagation conditions due to fading heavily disrupt the link reliability. This phenomenon can be contrasted using cooperative diversity techniques [1], where relays overhear the communication between two peers and assist them by repeating the data, or using time diversity techniques such as the Hybrid Automatic Repeat reQuest (HARQ) [2] protocol, consisting in the retransmission of data by the source in response to negative acknowledgements by the receiver. Both techniques, if individually applied, significantly improve the performance of the communication system. One open question is whether their combination would bring the sum of the singular improvements, or be only marginally beneficial.

In order to study the interaction of cooperation and HARQ in wireless networks we need to develop tools able to describe the system and its performance. In this work we use a performance analysis framework based on Finite State MarkovChains (FSMC) [3]. Markov models have been extensively used in the HARQ literature as a tool for performance prediction, both for Type I [4] and Type II [5], [6] point-topoint protocols. They have been widely employed also in the analysis of cooperative networks. In the cooperative networks literature, e.g., Markov chain models are employed to describe specific network topologies, as in [7] or [8] for multi-hop networks. In [9] FSMCs are developed to assist the design of cooperative systems with amplify-and-forward relaying.
Markov decision processes are used to assist relay selection [10], [11], [12].

The work in [13] is an example of Markov models used in schemes coupling ARQ and cooperation, which are the focus of this paper. It aims to optimize the number of cooperating relays in a specific declination of the ARQ protocol in an ad-hoc network, and achieves its objective using a two-states Markov process to model the transmission.

In Section II we introduce a simple three-node network composed by the source, the destination and a relay, and we define a deterministic communication protocol. The performance of the communication protocol can be evaluated using its representation by a Finite State Markov Chain, as done in Section III. This allows to predict the performance obtained for different values of the protocol parameters, thus enabling optimization. As the protocol gets more sophisticated, or as the number of retransmissions increases, the FSMC analysis becomes computationally heavy. In Section IV we show the existence of a probabilistic protocol which, while guaranteeing the same achievable performance of the deterministic protocol, is associated with a much more compact FSMC description, and hence simplifies the optimization process. This analysis, initially performed for the case of Type I HARQ decoding, is extended in Section V to the case of Type II HARQ decoding. Section VI presents numerical results obtained via Monte Carlo simulations, to verify the accuracy of the theoretical performance predictions.

\section{A DETERMINISTIC COMMUNICATION PROTOCOL}

Our example S-R-D network is composed of one source $(\mathrm{S})$, one relay (R), and one destination (D) [1]. The source $S$ needs to convey to the destination information Packet Data Units (PDUs) of fixed length, to which a Cyclic Redundancy Check (CRC) sequence is appended to enable the ARQ mechanism. The transmission happens in time-slots. The ARQ control messages are carried at the end of each time-slot from $\mathrm{D}$ to $\mathrm{S}$ and $\mathrm{R}$ by a feed-back channel which is assumed ideal. $\mathrm{S}$ and $\mathrm{R}$ use the same channel coding scheme. $\mathrm{R}$ is assumed to be working on the mode Demodulate-and-Forward (DMF) [14]. We first consider Type I HARQ: D attempts decoding of the PDU using only the last received coded packet. The extension to Type II HARQ is discussed in Section V. 


\begin{tabular}{|l|l|c|}
\hline State name & State definition & Next action \\
\hline 0 & $t_{S}=0, t_{R}=0, \mathrm{~W}=\mathrm{ACK}$ & $\mathrm{ST}$ \\
1 & $t_{S}=0, t_{R}=0, \mathrm{~W}=\mathrm{NACK}$ & $\mathrm{ST}$ \\
2 & $t_{S}=1, t_{R}=0, \mathrm{~W}=\mathrm{NACK}$ & $\mathrm{RRT}$ \\
3 & $t_{S}=1, t_{R}=1, \mathrm{~W}=\mathrm{NACK}$ & $\mathrm{RRT}$ \\
$\ldots$ & $\ldots$ & $\ldots$ \\
$N_{R}+2$ & $t_{S}=1, t_{R}=N_{R}, \mathrm{~W}=\mathrm{NACK}$ & $\mathrm{SRT}$ \\
$N_{R}+3$ & $t_{S}=2, t_{R}=0, \mathrm{~W}=\mathrm{NACK}$ & $\mathrm{RRT}$ \\
$N_{R}+4$ & $t_{S}=2, t_{R}=1, \mathrm{~W}=\mathrm{NACK}$ & $\mathrm{RRT}$ \\
$\ldots$ & $\ldots$ & $\ldots$ \\
$N_{S} N_{R}+N_{S}$ & $t_{S}=N_{S}, t_{R}=N_{R}-1, \mathrm{~W}=\mathrm{NACK}$ & $\mathrm{RRT}$ \\
\hline
\end{tabular}

TABLE I: Definition of states in the FSM of the transmitter

As soon as the first PDU is in the buffer, the source S broadcasts the channel coded packet, which is received by both $\mathrm{D}$ and R. D attempts decoding and issues the control message (ACK if decoding is successful, NACK if a decoding error is detected), received by both S and R. In case of ACK message, $\mathrm{S}$ proceeds with the transmission of a new information PDU. In case of NACK message, the protocol enters in the retransmission phase, where $\mathrm{R}$ and $\mathrm{S}$ retransmit in a deterministic order. A retransmission is performed every time that $\mathrm{D}$ issues NACK and the maximum number $N_{\max }$ of transmissions for the same PDU has not been reached yet. The retransmission order is determined by the following rules: 1) $R$ performs the first retransmission of the current information PDU; 2) $\mathrm{R}$ is allowed to retransmit $N_{R}$ consecutive times after each (re)transmission by S, after which the control of the channel goes back to $\mathrm{S}$; 3 ) $\mathrm{S}$ transmits the same PDU a maximum of $N_{S}$ times (this includes the first transmission). Each PDU is then allowed a maximum number of $N_{\max }=N_{S} \cdot N_{R}+N_{S}$ transmissions. $\mathrm{S}$ will proceed with the transmission of a new PDU any time that D issues an ACK, or after the $N_{\max }$-th failed transmission of the same PDU.

\section{A. Performance metrics definition}

Let $\nu$ be the total number of information PDUs sent by $\mathrm{S}$ during the operation time, $\sigma$ be the number of PDUs that have been ACK-ed by $\mathrm{D}$, and $\nu_{t}$ be the number of PDUs that have been transmitted exactly $t$ times. In order to express the performance of the protocol we consider the PDU Error Rate (PER), corresponding to the proportion of PDUs that were transmitted but never acknowledged by $\mathrm{D}$; and the average number of transmissions per PDU, denoted by $\bar{T}$ :

$$
\text { PER }=1-\frac{\sigma}{\nu}, \quad \bar{T}=\frac{1}{\nu} \sum_{t=1}^{N_{\max }} t \cdot \nu_{t} .
$$

\section{Protocol DESCRIPTION USING FSM AND FSMC}

The transmission protocol in the S-R-D network can be described using a FSM. The state of the FSM determines the action that is going to take place during the current time-slot, and the outcome of the action determines the transition to the next state.

The definition of the states for the considered protocol is given in Table I. At the beginning of the time-slot, let $t_{S}$ denote the number of times $\mathrm{S}$ has already transmitted the current

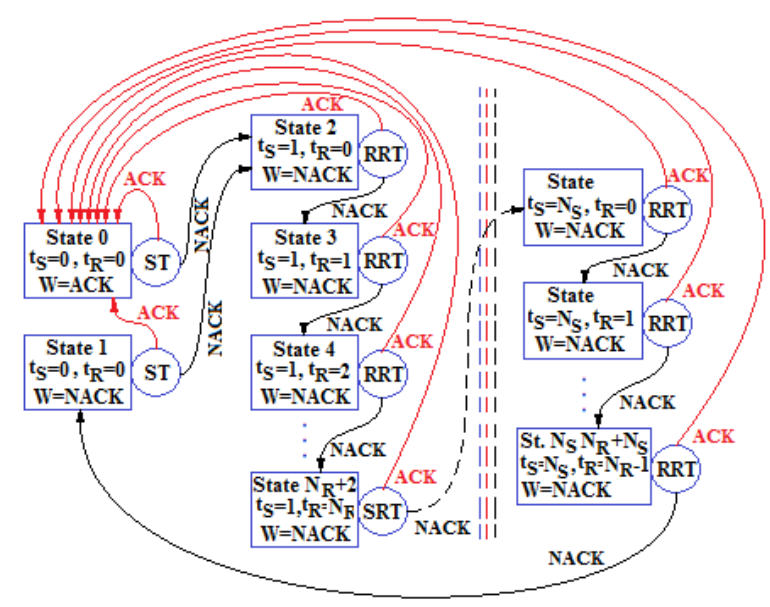

Fig. 1: FSM of the transmitter of the deterministic protocol

PDU; $t_{R}$ the number of times $\mathrm{R}$ has already transmitted the current PDU after the last transmission from S; and $W$ the last control message issued by $\mathrm{D}$. Each possible combination of the values of the parameters $t_{S}, t_{R}$ and $W$ defines a state. Notice that the combinations such that $\left\{1 \leq t_{S} \leq N_{S}, 1 \leq t_{R} \leq\right.$ $\left.N_{R}, W=\mathrm{ACK}\right\}$ are not possible, since $W=\mathrm{ACK}$ forces the transmission of a new PDU. Each state is associated with one of the possible actions: ST (Source Transmits a new PDU for the first time), SRT (Source Retransmits the current PDU) or RRT (Relay Retransmits the current PDU). The protocol starts in State 0 with the transmission of the first PDU.

The scheme of the possible state transitions in the FSM is given in Figure 1. Since the state transition depends only on the last input (corresponding to the last control message from D), the FSM has the Markov Chain property. The considered protocol can then be described by a Finite State Markov Chain (FSMC), with state transition matrix $P$, of size $\left(N_{S} \cdot N_{R}+N_{S}+1\right) \times\left(N_{S} \cdot N_{R}+N_{S}+1\right)$ :

$$
P=\left(\begin{array}{ccccccc}
1-\pi_{[1,0]} & 0 & \pi_{[1,0]} & 0 & \ldots & 0 & \ldots \\
1-\pi_{[1,0]} & 0 & \pi_{[1,0]} & 0 & \ldots & 0 & \ldots \\
1-\pi_{[0,1]} & 0 & 0 & \pi_{[0,1]} & \ldots & 0 & \ldots \\
\vdots & \vdots & \vdots & \vdots & \ddots & \vdots & \ddots \\
1-\pi_{[1,0]} & 0 & 0 & 0 & \ldots & \pi_{[1,0]} & \ldots \\
\vdots & \vdots & \vdots & \vdots & \ddots & \vdots & \ddots \\
1-\pi_{[0,1]} & \pi_{[0,1]} & 0 & 0 & \ldots & 0 & \ldots
\end{array}\right) .
$$

The element $[P]_{i j}$ represents the transition probability from State $i$ to State $j$. The symbol $\pi_{[1,0]}$ denotes the probability that $\mathrm{D}$ issues NACK after decoding the last copy received from $\mathrm{S}$, and $\pi_{[0,1]}$ denotes the probability that $\mathrm{D}$ issues NACK after decoding the last copy received from $\mathrm{R}$.

\section{A. Performance analysis using FSMC representation}

The steady state vector $\boldsymbol{p}$ is evaluated as the eigenvector associated to eigenvalue $\lambda=1$ of the transition matrix $P$. The steady state probability $p_{k}$ represents the fraction of time that the Markov Chain spends in state $k$ [3], and $p_{k}^{-1}$ represents 
the expectation of the recurrence time on state $k$. Observing that State 0 is visited only in case of correct acknowledgment of an information PDU, and that State 1 is visited only if a non-acknowledged PDU is dropped, the performance metrics (1) can be evaluated as functions of $\boldsymbol{p}$ as

$$
\operatorname{PER}=\frac{p_{1}}{p_{0}+p_{1}}, \quad \bar{T}=\frac{1}{p_{0}+p_{1}} .
$$

As a consequence, in order to predict the performance of the protocol, it is enough to derive its FSMC description. This requires to evaluate the probabilities $\pi_{[1,0]}$ and $\pi_{[0,1]}$ in (2), which depend on the channel coding scheme and on the propagation conditions on the channels S-R, R-D, and S-D. In the numerical examples presented in Section VI $\pi_{[1,0]}$ and $\pi_{[0,1]}$ are obtained via Monte-Carlo simulation.

Thanks to the structure of the matrix $P$, the steady state vector $\boldsymbol{p}$ can be also evaluated using a combinatorial approach. The states of the FSMC are visited in paths starting from State 0 or State 1 and going back to State 0 or State 1. Even if different paths may have different lengths, they share the property that State $i, \forall i>2$ is always reached from State $i-1$ (State 2 can be reached either by State 0 or 1 ). Define $c_{k}$ as the probability of accessing State $k$ for the first time during one of these paths. It is evaluated as

$$
\begin{gathered}
c_{0}=\sum_{t_{S}=1}^{N_{S}}\left(\sum_{t_{R}=0}^{N_{R}} \pi_{[1,0]}^{\left(t_{S}-\alpha\right)} \cdot \pi_{[0,1]}^{\left(t_{S}-1\right) N_{R}+t_{R}-1+\alpha} .\right. \\
\left.\cdot\left(1-\pi_{[1,0]}\right)^{\alpha} \cdot\left(1-\pi_{[0,1]}\right)^{\beta}\right), \\
c_{1}=\pi_{[1,0]}^{N_{S}} \cdot \pi_{[0,1]}^{N_{S} N_{R}}, \\
c_{\left(\left[t_{S}-1\right] N_{R}+t_{S}+t_{R}\right)}=\pi_{[1,0]}^{\left(t_{S}-\alpha\right)} \pi_{[0,1]}^{\left(t_{S}-1\right) N_{R}+t_{R}-1+\alpha},
\end{gathered}
$$

where $\alpha=1$ if $t_{R}=0$ and $\alpha=0$ otherwise; and $\beta=0$ if $t_{R}=0$ and $\beta=1$ otherwise. The steady state probability $p_{k}$ can be expressed as a function of the vector $c$ as

$$
\begin{aligned}
p_{k} & =\operatorname{Pr}(\text { being in steady state } k) \\
& =\operatorname{Pr}(\text { access state } k \mid \text { current path }) \\
& =\operatorname{Pr}\left(\text { access st. } k \mid \text { access st. } i \in\left\{0,1, \ldots, N_{S} N_{R}+N_{S}\right\}\right) \\
& =\frac{c_{k}}{\sum_{i=0}^{N_{S} N_{R}+N_{S}} c_{i}} .
\end{aligned}
$$

As $N_{\text {max }}$ gets larger, or as the protocol gets more sophisticated, the number of states in the FSMC increases. This happens, e.g., in switching $\mathrm{R}$ in Decode-and-Forward mode: whether $\mathrm{R}$ has the correct message from $\mathrm{S}$ needs to be encoded in the defition of the state, and this increases their number. This increases the complexity of the evaluation of the eigenvector of $P$, and hence on the performance prediction. This is the motivation for the simplified model in the following Section.

\section{State AGgREGATION AND PROBABILISTIC PROTOCOL}

Consider a FSMC $(\mathcal{S}, P)$ defined by a state set $\mathcal{S}=$ $\{0, \ldots, L\}$ and a transition matrix $P$. By aggregating multiple states of $(\mathcal{S}, P)$ it is possible to obtain a new $\operatorname{FSMC}(\mathcal{T}, Z)$ with state set $\mathcal{T}=\{0,1, \ldots, M\}$, with $M<L$, and transition matrix $Z$. We are interested in imposing the following constraint on the steady state vectors $\boldsymbol{p}$ and $\boldsymbol{z}$ of the FSMCs $(\mathcal{S}, P)$ and $(\mathcal{T}, Z)$, respectively. Let $I$ be the state in $(\mathcal{T}, Z)$ resulting from the aggregation of the set of states $\mathcal{I} \subseteq \mathcal{S}$ of $(\mathcal{S}, P)$. Let $J$ and $\mathcal{J}$ be defined similarly. Then the steady state probability $z_{I}$ of state $I$ is the sum of the steady state probabilities $p_{i}, i \in \mathcal{I}$

$$
z_{I}=\sum_{i \in \mathcal{I}} p_{i}
$$

The elements of the transition matrix $Z$ then become [15]:

$$
[Z]_{I J}=\frac{\sum_{i \in \mathcal{I}} p_{i}\left(\sum_{j \in \mathcal{J}}[P]_{i j}\right)}{\sum_{i \in \mathcal{I}} p_{i}} .
$$

In the FSMC described by Figure 1 and matrix (2) we are interested to aggregate the states associated with the same action, with the exception of States 0 and 1, which are left untouched. The states associated with the action RRT in Figure 1 are grouped to State 2 in the new FSMC, while the states associated with the action SRT are grouped in State 3. The result is a small FSCM with the following transition matrix

$$
Z=\left(\begin{array}{cccc}
1-\pi_{[1,0]} & 0 & \pi_{[1,0]} & 0 \\
1-\pi_{[1,0]} & 0 & \pi_{[1,0]} & 0 \\
1-\pi_{[0,1]} & \gamma \cdot \beta \pi_{[0,1]} & (1-\gamma) \pi_{[0,1]} & \gamma(1-\beta) \pi_{[0,1]} \\
1-\pi_{[1,0]} & 0 & \pi_{[1,0]} & 0
\end{array}\right) .
$$

The parameters $\gamma$ and $\beta$ in (10) take values according to (9). They also can be expressed using combinatorial approach: $\gamma$ represents the probability that $\mathrm{R}$ is performing its last retransmission before the channel goes back to $\mathrm{S}$; similarly, $\beta$ represents the probability that the maximum number of transmissions by $\mathrm{S}$ have already been performed. This implies that $\gamma$ and $\beta$ can also be evaluated using a combinatorial approach as

$$
\begin{aligned}
& \gamma=\frac{\pi_{[0,1]}^{N_{R}}}{\pi_{[0,1]}+\pi_{[0,1]}^{2}+\pi_{[0,1]}^{3}+\cdots+\pi_{[0,1]}^{N_{R}}}, \\
& \beta=\left(1+\sum_{t_{S}=1}^{N_{S}-1}\left(\frac{1}{\pi_{[1,0]}^{t_{S}} \cdot \pi_{[0,1]}^{t_{S} \cdot N_{R}}}\right)\right)^{-1} .
\end{aligned}
$$

Since, because of constraint (8), we have $z_{0}=p_{0}$ and $z_{1}=$ $p_{1}$, the steady state vector $\boldsymbol{z}$ of the matrix (10) can be used to evaluate the performance metrics (3) of the deterministic protocol.

The FSMC with transition matrix (10), illustrated by the scheme in Figure 2, can also be associated to a probabilistic protocol on the network S-R-D. The protocol works as follows. The first transmission of a new information PDU is performed by $\mathrm{S}$. In case retransmissions are needed the following rules are applied. 1) The first retransmission after any (re)transmission by $S$ is performed by $R$. 2) If $R$ is retransmitting, the next action is determined by the realization of a random variable with three possible values: $\mathrm{R}$ is allowed to retransmit in the 


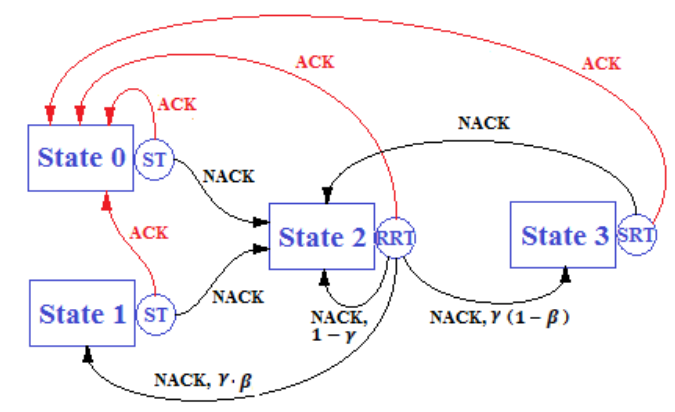

Fig. 2: Scheme of the simplified FSM on the transmitter

next time slot (probability $(1-\gamma)$ ); $\mathrm{S}$ is allowed to retransmit in the next time-slot (probability $\gamma(1-\beta)$ ); neither $\mathbf{R}$ or $\mathbf{S}$ are allowed to retransmit in the next time slot (probability $\gamma \cdot \beta$ ). In this last case $\mathrm{S}$ transmits a new PDU for the first time ${ }^{1}$.

Notice that the performance of the probabilistic protocol can be deduced using the steady state vector $z$ of the transition matrix (10). Both PER and $\bar{T}$ can be evaluated from (3) where $z_{0}=p_{0}$ and $z_{1}=p_{1}$, which is sufficient to guarantee that the performance of the probabilistic protocol in Figure 2 matches the deterministic protocol in Figure 1. Optimization of both protocols can be performed by predicting the performance for many possible combinations of the design parameters. The probabilistic protocol allows an easier optimization process while maintaining the same achievable performance, because the entire space of solutions can be explored by finding the steady state vector of the small matrix (10).

\section{CASE OF TYPE II HARQ}

In this Section we consider the protocol of Section II in presence of Type II HARQ decoder at D. While the Type I decoder only uses the most recently received packet, the Type II decoder combines all data relative to the same PDU received so far. In Section VI we present numerical results in a scenario where $\mathrm{S}$ and $\mathrm{R}$ send the same coded packet, and $\mathrm{D}$ decodes by Chase Combining [16] all the different copies. The analysis in this Section, however, holds also in the case of Incremental Redundancy Type II HARQ.

Since nothing changes in the protocol rules, its description via the FSMC in Figure 1 is still valid. In case of Type II HARQ, however, the transition matrix takes the form:

$P_{c}=\left(\begin{array}{ccccccc}1-\pi_{[1,0]} & 0 & \pi_{[1,0]} & 0 & \cdots & 0 & \cdots \\ 1-\pi_{[1,0]} & 0 & \pi_{[1,0]} & 0 & \cdots & 0 & \cdots \\ 1-\pi_{[1,1]} & 0 & 0 & \pi_{[1,1]} & \cdots & 0 & \cdots \\ \vdots & \vdots & \vdots & \vdots & \ddots & \vdots & \ddots \\ 1-\pi_{\left[1, N_{R}\right]} & 0 & 0 & 0 & \cdots & \pi_{\left[1, N_{R}\right]} & \cdots \\ \vdots & \vdots & \vdots & \vdots & \ddots & \vdots & \ddots \\ 1-\pi_{\left[N_{S}, N_{S} N_{R}\right]} \pi_{\left[N_{S}, N_{S} N_{R}\right]} & 0 & 0 & \cdots & 0 & \cdots\end{array}\right)$

\footnotetext{
${ }^{1}$ The probabilistic protocol can work in absence of a centralized unit if the transmitting nodes exchange the random generator seed at the beginning of the operation interval, and draw locally the realizations of the random variable.
}

where $\pi_{[A, B]}$ is the probability that $\mathrm{D}$ fails decoding the current PDU, based on $A$ copies received from $\mathrm{S}$ and $B$ copies received from $\mathrm{R}$. In the numerical examples presented in Section VI the parameters $\pi_{[A, B]}$ are evaluated via Monte Carlo simulation.

As done for Type I decoding, we can consider the probabilistic protocol represented in Figure 2 in the case of Type II decoding also. In Section IV we have seen how the performance of the probabilistic protocol for Type I decoding can be predicted by steady state analysis of the small matrix $Z$ obtained by state aggregation on the matrix $P$. As done in the case of Type I decoder, we aggregate the states of the FSMC associated to the events RRT or SRT. The simplified FSMC model is obtained using (9) and its corresponding transition matrix can be expressed as:

$$
Z_{c}=\left(\begin{array}{cccc}
1-\pi_{[1,0]} & 0 & \pi_{[1,0]} & 0 \\
1-\pi_{[1,0]} & 0 & \pi_{[1,0]} & 0 \\
1-\pi_{[R F]} & \gamma \cdot \beta \pi_{[R F]} & (1-\gamma) \pi_{[R F]} & (1-\beta) \pi_{[R F]} \\
1-\pi_{[S F]} & 0 & \pi_{[S F]} & 0
\end{array}\right)_{(14)}
$$

As before, $\gamma$ is the probability that $\mathrm{R}$ is performing its last retransmission before the channel goes back to $\mathrm{S}$ and $\beta$ is the probability that the maximum number of transmissions by $S$ have already been performed. The parameters $\pi_{[R F]}$ and $\pi_{[S F]}$ represent the average probability of failure in decoding at $\mathrm{D}$ after the reception of a retransmission from $\mathrm{R}$ and from $\mathrm{S}$, respectively. The average value accounts for the fact that the probability of acknowledgment of a PDU does not depend only on whether is R or S performed the most recent transmission, but also on how many copies D had already received. This implies that the FSM describing the probabilistic protocol depicted in Figure 2 does not satisfy, for Type II decoding, the Markov property, and hence cannot be associated to a FSMC. Nonetheless, as shown by the simulation results in Section VI, using the Type II probabilistic protocol with parameters $\gamma$ and $\beta$ resulting from state aggregation of the matrix $P_{c}$ still allows to obtain very close performance to the case of the deterministic Type II protocol. This suggests that the use of a probabilistic protocol does not determine a significant performance loss.

\section{NUMERICAL RESULTS}

In this Section we present simulation results to confirm that the FSMC analysis is able to predict the performance of the system. We consider an S-R-D network, when the Relay is located close to the Source at distance $d_{S R}=0.15 \cdot d_{S D}$. The channels S-D, S-R and R-D are Gaussian channels with noise density $N_{0}$ suffering from block-fading. We consider the 16-QAM modulation scheme and a convolutional code with rate $R_{c}=1 / 3$. The transmitting nodes $\mathrm{S}$ and $\mathrm{R}$ use the same energy per symbol $E_{s}$. The parameters of deterministic protocol are chosen $N_{S}=2$ and $N_{R}=1$, while the values of parameters $\gamma$ and $\beta$ for the probabilistic protocol are evaluated from the simplification analysis of deterministic protocol. Figure 3 shows the comparison of the PER as a function of receive $E_{b} / N_{0}$ on the channel S-D, achieved with 


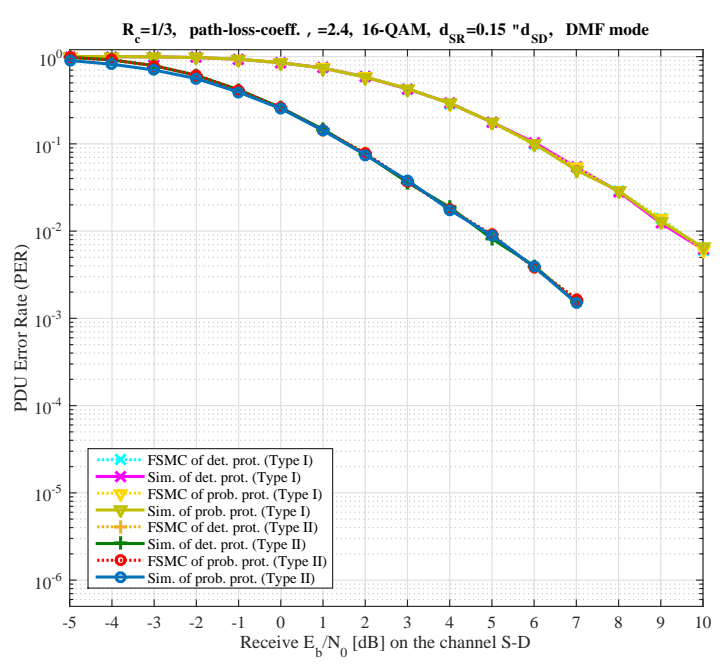

Fig. 3: PER achieved with deterministic and probabilistic protocol

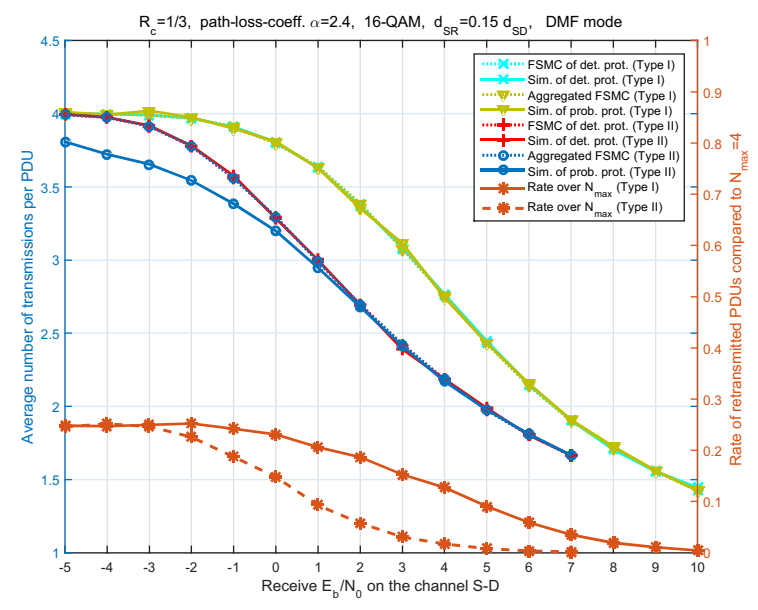

Fig. 4: $\bar{T}$ achieved with deterministic and probabilistic protocol

the deterministic and probabilistic protocols for the case of Type I HARQ and Type II HARQ with Chase Combining. As expected, the Type II decoder greatly outperforms Type I. The prediction of the FSMC models are very accurate with respect to the simulated performances. The aggregated FSMC model is accurate also for the case of the probabilistic protocol with Type II decoder. Figure 4, left y axis, depicts $\bar{T}$ predicted and simulated. Even if it is not accurate for low values of receive $E_{b} / N_{0}$, the aggregated FSMC for the Type II decoder provides a good prediction in the interesting SNR region. Notice in the probabilistic protocol no maximum number of transmissions per PDU is imposed. In Figure 4, right y axis, we show the proportion of PDUs that get transmitted more than $N_{\max }$ times, i.e. exceed the maximum number of retransmissions allowed by the deterministic protocol. As expected, this proportion decreases as the channel improves, and is much restrained in the case of Type II decoder.

\section{CONCLUSIONS}

This work considers the problem of performance analysis in cooperative wireless networks with HARQ. Using an example on a S-R-D network we show that Finite State Markov Chains (FSMCs) are able to efficiently describe the communication protocol and predict its performance. However, as the protocol grows more complex, the approach lacks scalability. This problem is solved by simplification of the FSMC analysis via state aggregation. The simplified FSMC is very compact, and allows fast prediction of the performance. Moreover, we can associate the simplified FSMC with a probabilistic protocol that can be optimized easily by adjusting two parameters that bring the best performance. The analysis strategy developed here can be applied in topologies with more nodes (e.g., in presence of multiple relays), where the simplified model will be most beneficial in reducing the complexity.

\section{REFERENCES}

[1] A. Nosratinia, T. E. Hunter, and A. Hedayat, "Cooperative communications in wireless networks," IEEE Communications Magazine, vol. 42, no. 10, pp. 74-80, 2004.

[2] H. A. Ngo and L. Hanzo, "Hybrid Automatic-Repeat-reQuest systems for cooperative wireless communications," IEEE Communications Surveys \& Tutorials, vol. 16, no. 1, 2014.

[3] R. G. Gallager, Stochastic Processes, Theory for Applications. Cambridge University Press, 2014.

[4] Q. Zhang, T. F. Wong, and J. S. Lehnert, "Performance of a Type-II Hybrid ARQ protocol in slotted DS-SSMA packet radio systems," IEEE Transactions on Communications, vol. 47, no. 2, 1999.

[5] M. Zorzi and R. R. Rao, "Throughput analysis of ARQ selective-repeat protocol with time diversity in Markov channels," in Proc. of IEEE Global Telecommunications Conference (GLOBECOM), 1995.

[6] L. Badia, M. Levorato, and M. Zorzi, "Markov analysis of selective repeat Type II Hybrid ARQ using block codes," IEEE Transactions on Communications, vol. 56, no. 9, 2008.

[7] S. A. Hassan and M. A. Ingram, "A quasi-stationary Markov chain model of a cooperative multi-hop linear network," IEEE Transactions on Wireless Communications, vol. 10, no. 7, 2011.

[8] S. A. Hassan, "Performance analysis of cooperative multi-hop strip networks," Wireless Personal Communications, vol. 74, no. 2, 2014.

[9] Y. Luo, R. Zhang, L. Cai, and S. Xiang, "Finite-state Markov modelling for wireless cooperative networks," IET Networks, vol. 3, no. 2, 2014.

[10] G. N. Shirazi, P. Kong, , and C. Tham, "Markov decision process frameworks for cooperative retransmission in wireless networks," in Proc. of the IEEE Wireless Communications and Networking Conference (WCNC), Budapest, Hungary, 2009.

[11] F. Babich, A. Crismani, and L. Hanzo, "Relay selection schemes relying on adaptive modulation and imperfect channel knowledge for cooperative networks," in Proc. IEEE Int. Conf. on Communications (ICC), 2012.

[12] Y. Li, P. Wang, D. Niyato, and W. Zhuang, "A hierarchical framework of dynamic relay selection for mobile users and profit maximization for service providers in wireless relay networks," Wireless Communications and Mobile Computing, vol. 14, no. 12, 2014.

[13] M. Dianati, X. Ling, K. Naik, and X. Shen, "A node-cooperative ARQ scheme for wireless ad hoc networks," IEEE Transactions on Vehicular Technology, vol. 55, no. 3, 2006.

[14] Y. Yang, H. Hu, J. Xu, and G. Mao, "Relay technologies for Wimax and LTE-Advanced mobile systems," IEEE Communications Magazine, vol. 47, no. 10, 2009.

[15] H. D. Sterck, T. A. Manteuffel, S. F. Mccormick, Q. Nguyen, and J. Ruge, "Multilevel adaptive aggregation for Markov chains, with application to web ranking," SIAM Journal on Scientific Computing, vol. 30 , no. $5,2008$.

[16] D. Chase, "Code combining: A maximum-likelihood decoding approach for combining an arbitrary number of noisy packets," IEEE Transactions on Communications, vol. 33, no. 5, 1985. 